\section{A E E T}

ASOCIACIÓN ESPAÑOLA DE ECOLOGÍA TERRESTRE

\title{
Cambios en los límites de distribución de especies arbóreas como consecuencia de las variaciones climáticas
}

\author{
L. Matías ${ }^{1, *}$ \\ (1) Biological and Environmental Sciences, School of Natural Sciences, University of Stirling, Stirling, FK9 4LA, UK
}

*Autor de correspondencia: L. Matías [luis.matiasresina@stir.ac.uk]

> Recibido el 28 de septiembre de 2012, aceptado el 6 de noviembre de 2012

\begin{abstract}
Matías, L. (2012). Cambios en los límites de distribución de especies arbóreas como consecuencia de las variaciones climáticas. Ecosistemas 21(3):91-96. Doi.: 10.7818/ECOS.2012.21-3.12

Los cambios registrados en el clima durante las últimas décadas están afectando a la distribución y dinámica poblacional de las especies a una escala global, induciendo cambios en la fenología, en el crecimiento o en las interacciones bióticas. Estas alteraciones se espera sean más patentes en los márgenes de la distribución de las especies, donde pueden provocar expansiones o contracciones de rango. En este trabajo, revisaré los últimos avances en el estudio de la dinámica poblacional de especies arbóreas en sus límites altitudinales y latitudinales y cómo su distribución se ve afectada por los cambios en el patrón climático. Además, analizaré cómo los efectos de las variaciones en el clima pueden actuar conjuntamente con otros efectos indirectos, como es el caso de las alteraciones en algunas interacciones bióticas, entre las que destacan los cambios de distribución y presión de plagas, patógenos o herbívoros.
\end{abstract}

Palabras clave: cambio climático, interacciones bióticas, sequía, temperatura.

Matías, L. (2012). Changes in the distribution limits of tree species as a consequence of climate variations. Ecosistemas 21(3):91-96. Doi.: 10.7818/ECOS.2012.21-3.12

Changes in climate registered during the last decades are affecting the distribution and population dynamics of plant species at a global scale, inducing changes in phenology, growth and biotic interactions. These alterations are expected to be most evident at species range edges, where they can result in range expansions or contractions. In this paper, I will review the last knowledge in the study of the population dynamics of tree species at their altitudinal and latitudinal range limits, and how their distribution might be affected by the alteration of the climatic pattern. In addition, I will analyse how climate change may induce indirect effects on plant populations through the alteration of biotic interactions, such as the modification of the intensity and distribution of pests, pathogens and herbivores.

Keywords: biotic interactions, climate change, drought, temperature.

\section{Introducción}

Las especies vegetales no muestran una distribución global sino que se restringen a unos determinados rangos geográficos donde los factores históricos les permitieron formarse o colonizar, y donde actualmente existen una serie de condiciones bióticas y abióticas aptas para el crecimiento y la reproducción (Woodward 1987). Sin embargo, los importantes cambios en el clima que se están registrando a escala global (IPCC 2007) están alterando estas condiciones, induciendo cambios en la fenología, crecimiento o en la dinámica poblacional de muchas especies (Nemani et al 2003; Parmesan 2006; Parmesan y Yohe 2003), así como en las interacciones bióticas, ya sea por la alteración de éstas o por la aparición de otras nuevas (Hughes 2000; Suttle et al. 2007). A menudo, estos cambios son mucho más evidentes en los límites de distribución de las especies, donde las condiciones ecológicas ya se encuentran próximas a los límites de tolerancia y donde es previsible que las consecuencias sean mucho más severas (Hampe y Petit 2005). A nivel poblacional, estos cambios inducen alteraciones en los rangos de distribución, lo que puede tener unas importantes consecuencias para la estructura y el funcionamiento de las comunidades o para la conservación de las especies amenazadas (Parmesan y Yohe 2003; Walther 2010).
La expansión del rango de las especies está controlada por diferentes factores en sus límites meridional y septentrional. Así, mientras que en las zonas más próximas a los polos y de mayor altitud las bajas temperaturas son el factor limitante, en las zonas bajas o más cercanas al Ecuador la distribución se limita por una combinación de altas temperaturas y sequía (Woodward 1987). La principal diferencia entre ambas es que mientras que se espera que las bajas temperaturas que limitan en uno de los extremos sean menos severas durante las próximas décadas, la limitación por altas temperaturas y la sequía serán mucho más intensas en el otro extremo. La analogía entre altitud y latitud nos lleva a hablar, de forma simplificada de un frente de avance, tanto en los límites superiores de las zonas montañosas como en el límite norte de la distribución, donde las que las condiciones ecológicas dejan de ser limitantes, y de un frente de retroceso en las cotas más bajas de las montañas o en el límite meridional de la distribución, donde se esperan unas condiciones cada vez más adversas. Si los cambios en las condiciones climáticas inducen alteraciones en los límites de distribución de las especies, podemos diferenciar tres tipos de cambio de distribución en función de las zonas que se vean afectadas: (1) expansión de rango: si se aumenta la distribución por el frente de avance, pero no se producen cambios en el frente de retroceso; (2) contracción de rango: si se reduce el área ocupada 
por la especie en el frente de retroceso y no hay cambios en el frente de avance; y (3) desplazamiento de rango: si se produce tanto un aumento de área en el frente de avance como una disminución en el frente de retroceso.

Los cambios en el clima pueden afectar a la dinámica de los ecosistemas mediante la introducción o extinción de especies o mediante cambios en la fenología, la fisiología o en la dinámica poblacional de muchas de sus especies (Parmesan 2006).Cabe esperar que las interacciones bióticas, tanto las mutualistas como las antagonistas, se vean alteradas o que aparezcan otras nuevas. Esta alteración puede modificar la respuesta individual de las especies a los cambios ambientales o generar efectos no aditivos que pueden llegar a tener importantes consecuencias sobre la dinámica del ecosistema (Bligler et al. 2006; Tylianakis et al. 2008; Van der Putten et al. 2010). Por tanto, es muy importante tener en cuenta los posibles cambios en las interacciones bióticas a la hora de estudiar las consecuencias del cambio climático sobre determinadas especies.

Dada la alta importancia de los cambios que se están produciendo durante las últimas décadas a nivel de ecosistema, en esta revisión discutiré los posibles efectos que los cambios en el clima pueden inducir en la distribución de las especies arbóreas no tropicales, resaltando la importancia de los límites del rango. En concreto, examinaré los cambios observados en los frentes de avance y de retroceso, así como en las interacciones bióticas que pueden afectar a la dinámica poblacional de estas especies y las posibles tendencias poblacionales que cabe esperar para las próximas décadas.

\section{Frente de avance}

Tanto la dinámica poblacional como el comportamiento de los individuos de las especies leñosas en el límite arbóreo están fuertemente determinados por la temperatura (Körner 2012). Por tanto, es lógico esperar cambios en el crecimiento, en la tasa reproductiva o en el reclutamiento ante las previsiones de calentamiento esperadas para las próximas décadas a escala global. Estos cambios serán mucho mas evidentes en el frente de avance (tanto altitudinal como latitudinal) ya que el principal factor limitante aquí suelen ser las bajas temperaturas (Fig. 1). A corto plazo, estos cambios en el clima pueden alterar la tasa reproductiva, el reclutamiento, el crecimiento o la estructura de la población, mientras que a largo plazo pueden inducir cambios en el rango de distribución de las especies.

\section{Reproducción}

La producción de semillas esta fuertemente determinada por la temperatura en el frente de avance de las especies arbóreas (Pohtila 1980; Almqvist et al. 1998; Juntunen y Neuvonen 2006). A medida que nos acercamos al límite forestal, la producción de semillas por unidad de área normalmente se reduce debido a cuatro razones principalmente: (1) aclaramiento del bosque y menor tamaño de los árboles; (2) mayores intervalos entre años buenos para la producción de semillas; (3) menor cantidad de frutos/semillas por biomasa arbórea; y (4) menor viabilidad de las semillas producidas (Körner 2012). Por lo tanto, un aumento global de la temperatura como el esperado para las próximas décadas podría tener un claro efecto sobre los puntos 2, 3 y 4 y, a medio-largo plazo, sobre el 1 . De hecho, ya se ha registrado un aumento significativo en la producción de semillas en el límite norte de la distribución de algunas especies, como es el caso de Pinus sylvestris y Picea abies en el norte de Finlandia (Holtmeier y Broll 2011; Juntunen y Neuvonen 2006; Kullman 2007), lo que hace pensar que la limitación de semillas no sea un factor determinante para el avance del límite forestal en las próximas décadas.

\section{Reclutamiento y crecimiento}

En el límite forestal, se ha demostrado que las especies arbóreas responden de una forma más evidente al calentamiento que al enfriamiento de las condiciones ambientales (Kullman 1990), y una de las respuestas más claras es el establecimiento de nuevas plántulas y la densificación del banco de juveniles (Juntunen y Neuvonen 2006). Puesto que el reclutamiento es considerado como el factor más determinante en el control de las dinámicas poblacionales en el límite forestal (Rickebusch et al. 2007), el aumento que se ha detectado en la tasa de reclutamiento en algunas especies arbóreas a lo largo de su límite altitudinal o latitudinal de distribución (Harsch et al. 2009; Juntunen y Neuvonen 2006; Kullman 2002, 2010; Stöcklin y Körner 1999) puede indicar un avance del rango. Sin embargo, existe una elevada heterogeneidad espacial incluso dentro de la misma especie, a menudo controlada por factores locales como la topografía o la presencia de otras especies.

Al igual que la reproducción y el establecimiento, tanto el crecimiento primario como el secundario están fuertemente determinados por la temperatura en el límite forestal (Körner 1999; Mäkinen et al. 2000; Rickebusch et al. 2007). Durante las últimas décadas se han indicado numerosos ejemplos de aumentos de crecimiento directamente relacionados con una tendencia al alza de las temperaturas (MacDonald et al. 1998; Mathisen y Hofgaard 2011; Salminen y Jalkinen 2007), aportando evidencias de que la limitación por bajas temperaturas podría jugar un papel cada vez menos importante en un futuro próximo en la dinámica poblacional del límite forestal. Sin embargo, es necesario tener en cuenta que un calentamiento del clima puede conllevar un aumento de la aridez, incluso en bosques boreales, lo que podría llegar a contrarrestar el efecto positivo del aumento de temperatura en algunos casos (Lloyd y Fastie 2002).

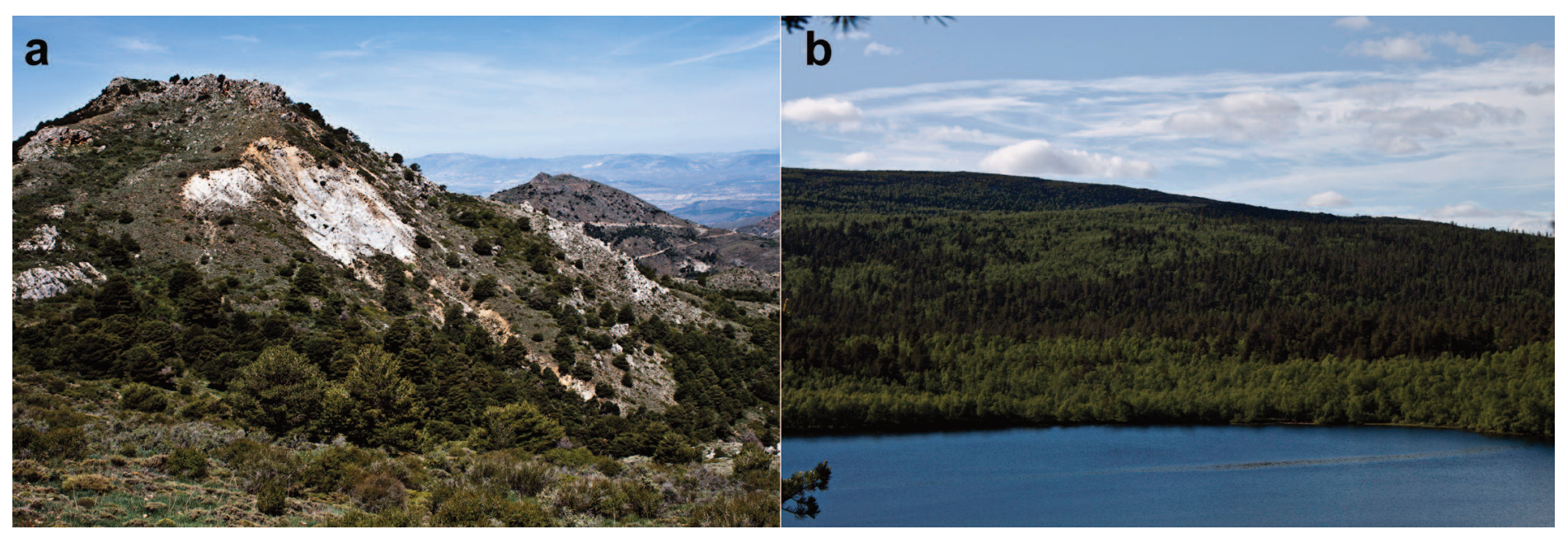

Figura 1. Límite altitudinal de del pino silvestre (Pinus sylvestris L.) en los extremos de su distribución latitudinal: a) límite meridional (Sierra Nevada, España) y b) límite septentrional (Kevo, Finlandia). Foto: Luis Matías. 
Tabla 1: Casos documentados de A: expansiones recientes de rango en altitud de especies forestales y B: eventos de mortalidad masiva asociados a sequia o altas temperaturas en los límites inferior o meridional de la distribución. Modificado de Allen et al. (2010) y de Walther (2003).

A. Expansiones de rango

\begin{tabular}{|c|c|c|c|c|}
\hline País & Periodo & Especie & Cambio altitudinal & Referencia \\
\hline Canadá & 150 años & Picea glauca & $10-20 \mathrm{~m}$ & Szeicz y MacDonald (1995) \\
\hline Rusia & Desde 1920 & Larix sibrica & $20-30 \mathrm{~m}$ & Shiyatov (2000) \\
\hline Nueva Zelanda & 60 años & Nothofagus ssp. & $5-8 \mathrm{~m}$ & Wardle y Coleman (1992) \\
\hline Suecia & 50 años & $\begin{array}{l}\text { Betula pubescens, Sorbus aucuparia, } \\
\text { Picea abies, Pinus sylvestris, } \\
\text { Salix ssp., Acer platanoides }\end{array}$ & $120-375 m$ & Kullman (2002) \\
\hline España & Desde 1955 & Fagus sylvatica & $70 \mathrm{~m}$ & Peñuelas y Boada (2003) \\
\hline Bulgaria & Desde 1970 & Pinus peuce & $130 \mathrm{~m}$ & Meshinev et al. (2000) \\
\hline Finlandia & Desde 1983 & Pinus sylvestris, Picea abies & & Juntunen et al. (2002) \\
\hline \multicolumn{5}{|c|}{ B. Mortalidad masiva } \\
\hline País & Periodo & Especie & Causa mortalidad & Referencia \\
\hline Suiza & $1960-2004$ & Pinus sylvestris & Sequía & $\begin{array}{l}\text { Bigler et al. (2006); Dobbertin et al. } \\
\text { (2007); Wermelinger (2008) }\end{array}$ \\
\hline Senegal & $1972-1973$ & Acacia senegal, Guiera senegalensis & Sequía & Poupon (1980) \\
\hline EEUU & 1983-2004 & Pinus ssp., Abies ssp. & Sequía & van Mantgem y Stephenson (2007) \\
\hline EEUU & $1985-1995$ & Pinus flexilis & Sequía & Millar et al. (2007) \\
\hline Arabia Saudí & 1990 & Juniperus procera, J. excelsa & Sequía & Fisher y Gardner (1995) \\
\hline Austria & $1990-1996$ & Pinus sylvestris, P.nigra & Sequía & Cech y Tomiczeck (1996) \\
\hline Italia & 1992 & Pinus sylvestris & Sequía & Minerbi (1993) \\
\hline Argentina & 1998-1999 & Nothofagus dombeyi & Sequía & Suarez et al. (2004) \\
\hline Grecia & 2000 & Pinus brutia & Sequía & Körner et al. (2005) \\
\hline Turquía & $2002-2007$ & $\begin{array}{l}\text { Quercus ssp., Juniperus ssp., Pinus nigra, } \\
\text { P. sylvestris, Abies ciclicia }\end{array}$ & Sequía & Semerci et al. (2008) \\
\hline Marruecos & $2002-2008$ & Cedrus atlantica & Sequía & El Abidine (2003); Adil (2008) \\
\hline Francia & $2003-2008$ & Pinus sylvestris & Sequía, temperatura & $\begin{array}{l}\text { Vennetier et al. (2007); } \\
\text { Thabeet et al. (2009) }\end{array}$ \\
\hline España & 2004-2006 & Pinus sylvestris, P.nigra & Sequía & Navarro-Cerrillo et al. (2007) \\
\hline
\end{tabular}

\section{Avance del límite forestal}

El incremento de $3^{\circ} \mathrm{C}$ previsto para finales de este siglo por los principales modelos climáticos (IPCC 2007) implica que, al menos en teoría, un aumento altitudinal del límite forestal de $500 \mathrm{~m}$ podría ser plausible (Grace et al. 2002). De hecho, durante las dos últimas décadas se han encontrado numerosas evidencias de un aumento tanto en altitud como en latitud del límite arbóreo (Tabla 1). Sin embargo, aunque este aumento de $500 \mathrm{~m}$ podría ser posible en algunas zonas y para determinadas especies (ver por ejemplo Kullman 2002; Kullman y Öberg 2009), este escenario no es real a una escala de paisaje, ya que los casos que se han descrito de avance rápido están limitados a unas zonas muy concretas con condiciones topográficas y microclimáticas favorables. Además, los cambios latitudinales de rango ocurren a distancias hasta mil veces mayores que en el caso de altitud, por lo que es muy probable que las limitaciones en la dispersión jueguen un papel muy importante en este caso (Jump et al. 2009). A pesar de estas evidencias, basándose en registros de cambios anteriores en el clima, encontramos que los cambios en la posición del límite forestal siempre tienen un retraso respecto a los cambios en el clima de al menos 50 años debido, entre otros factores, al desacople entre años buenos para la reproducción y para el establecimiento, el largo tiempo necesario para que un árbol establecido más allá del límite llegue a ser reproductor, o la presencia de eventos excepcionalmente fríos a pesar de una tendencia generalizada de crecimiento (Körner 2012). Por otro lado, a la hora de predecir los cambios futuros hay que tener en cuenta otra serie de factores además de los estrictamente climáticos que pueden favorecer (como la existencia de árboles aislados que actúen como núcleos de dispersión o procesos de facilitación por la vegetación existente o las condiciones topográficas) o impedir (como competencia o herbivoría) el avance del límite forestal (Slot et al. 2005; Stöcklin y Körner 1999).

\section{Frente de retroceso}

Los cambios en la dinámica poblacional de las especies arbóreas no son tan rápidos en el frente de retroceso como en el frente de avance debido principalmente a la alta longevidad y resiliencia de estas especies, que les hace persistir en una zona a pesar de que las condiciones ambientales sean adversas (Hampe y Jump 2011). Sin embargo, ya se han detectado numerosas evidencias sobre la posible contracción del rango de distribución de las especies arbóreas debido al aumento de las temperaturas y de la sequía. En esta sección comentaré algunas de ellas.

Uno de los principales procesos que se están viendo afectados en el frente de retroceso es el reclutamiento. Las fases de plántula y juvenil son las más sensibles a la sequía en el ciclo ontogenético de las especies arbóreas, al disminuir el crecimiento y elevar la tasa de mortalidad, pudiendo llegar incluso a un colapso total del reclutamiento durante los veranos más secos (Castro et al. 2004; Mendoza et al. 2009). Si la frecuencia de estos eventos de sequía extrema se incrementa según lo esperado por los principales modelos climáticos (IPCC 2007), aquellas poblaciones que se encuentren en el frente de retroceso solo podrán reclutar nuevos individuos 
durante los años excepcionalmente lluviosos (Matías et al. 2012), que a su vez también pueden reducir su frecuencia. A medio plazo, una disminución en el reclutamiento inducirá poblaciones envejecidas y con una estructura de edad desproporcionada. De igual forma, el crecimiento también se ve afectado de forma negativa por la sequía y el aumento de las temperaturas. La disminución en la precipitación no solo es capaz de reducir el crecimiento de forma progresiva (Bogino et al. 2009; Sánchez-Salguero et al. 2012a, b), sino que además la sucesión de varios años de sequía moderada reduce sensiblemente la capacidad de recuperación después de un evento extremo (Linares et al. 2012; Martínez-Vilalta et al. 2012a, b). Además, una reducción en el crecimiento durante varios años consecutivos lleva a un debilitamiento general de los individuos, que puede inducir importantes consecuencias a nivel poblacional como la mayor sensibilidad a patógenos o sequías extremas $y$, en ultima instancia, a la muerte del individuo (Bigler et al. 2006). Como consecuencia, en los últimos años se han detectado eventos de mortalidad masiva en poblaciones de árboles a escala global (Tabla 1) que, unidos a la baja tasa de reclutamiento, pueden llevar a contracciones repentinas del rango de distribución.

\section{Cambios en las interacciones bióticas}

Como hemos visto anteriormente, las actuales predicciones climáticas apoyan firmemente un cambio en la distribución de las especies. Sin embargo, cada vez hay mas evidencias de que una alteración en el clima también tendrá importantes consecuencias sobre las interacciones bióticas (Tylianakis et al. 2008; Van der Putten et al. 2010). Los cambios en las condiciones climáticas no tienen por qué afectar por igual a todas las especies del ecosistema, lo que puede inducir tanto cambios en las interacciones bióticas existentes como la aparición de otras. Uno de los cambios más evidentes como respuesta a las variaciones de temperatura es el incremento de plagas y patógenos, especialmente de insectos y hongos (Bale et al. 2002; Neuvonen et al. 1999; Sikström et al. 2011). De forma general, se espera que el ataque a las especies arbóreas sea cada vez mas intenso debido tanto a explosiones demográficas de las plagas y patógenos como a un aumento de su virulencia en respuesta a un incremento de temperatura (Dobbertin et al. 2007; Fabre et al. 2011; Juntunen y Neuvonen 2006), así como unas mayores consecuencias de los ataques debido al debilitamiento de los árboles inducido por la sequía en determinadas zonas (Bigler et al. 2006). Además, unos inviernos más suaves favorecen la expansión de las especies plaga en altitud y en latitud (Bale et al. 2002; Hódar et al. 2012), lo que permite la aparición de nuevas interacciones entre especies que no han coexistido hasta la fecha (ver por ejemplo la interacción entre Thaumetopoea pytiocampa y Pinus sylvestris en Hódar et al. 2003). De forma similar, el aumento de la temperatura y humedad previsto en el límite norte de las especies arbóreas puede favorecer la dispersión y el ataque de hongos patógenos (Sikström et al. 2011; Stöcklin y Körner 1999), lo que puede llegar a modular el posible avance del límite forestal.

Los herbívoros pueden ejercer también un efecto muy importante sobre el control de la dinámica poblacional de las especies en el límite forestal, tanto el norte como en el sur de la distribución (Cairns y Mohen 2004; Herrero et al. 2012). Éstos pueden interactuar con los efectos producidos por los cambios en el clima $(\mathrm{He}-$ rrero et al. 2012), por lo que se debe tener en cuenta la densidad de ungulados (tanto domésticos como salvajes) a la hora de predecir los cambios en el límite forestal bajo un escenario de cambio climático. Además, otros factores como la facilitación pueden modular en gran medida la respuesta de las especies arbóreas a los cambios ambientales, favoreciendo el reclutamiento a pesar de las condiciones adversas (Gómez-Aparicio et al. 2004). Por último, debemos tener en cuenta las posibles consecuencias de la competencia con otras especies vegetales, tanto las existentes en aquellas zonas en las que el rango se expande, como aquellas (invasoras o no) que se establecen en el rango actual de las especies arbóreas.

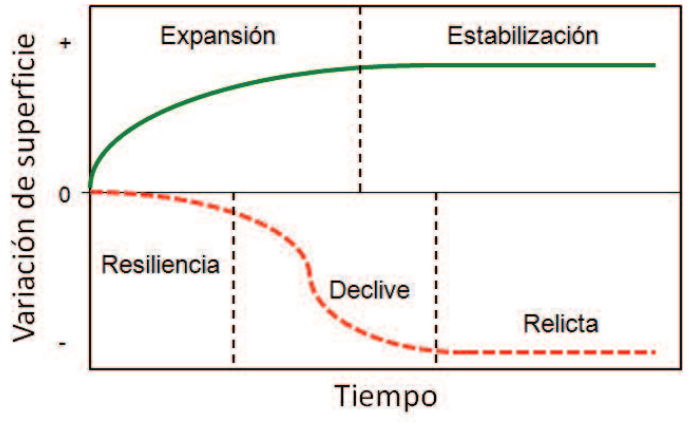

Figura 2. Cambios esperados en el área ocupada por las especies forestales en los límites norte (línea verde continua) y sur (línea roja discontinua) de su distribución bajo un escenario de cambio climático a lo largo del tiempo. Valores positivos representan expansiones de rango, mientras que valores negativos indican contracciones respecto a la superficie actualmente ocupada. Modificado de Matías y Jump (2012).

\section{Conclusiones}

Como hemos visto, los recientes cambios registrados en el clima pueden alterar, o están ya alterando, el límite de distribución de las especies forestales. Aunque las respuestas son a menudo específicas para cada especie y dependen de muchos factores que pueden estar interaccionando de forma conjunta, se pueden extraer algunas generalidades. Según el modelo propuesto por Matías y Jump (2012), y a tenor de lo comentado anteriormente, el comportamiento de las poblaciones esperado en el frente de avance y de retroceso para las próximas décadas es claramente distinto. En el frente de avance podemos esperar una fase inicial de expansión poblacional más o menos rápida debido al incremento de reproducción y crecimiento en respuesta al aumento de temperatura, seguida de una fase de estabilización una vez alcanzadas las condiciones óptimas de la especie o se haya ocupado todo el nicho potencial (Fig. 2). Al contrario, en el frente de retroceso cabría esperar una primera fase de resiliencia en la que se mantienen las poblaciones relativamente estables debido a la longevidad de las especies arbóreas pese a que las condiciones ya no son óptimas, seguida de una fase rápido declive poblacional una vez que se superan los límites para la supervivencia de los individuos, pudiendo producirse eventos de mortalidad masiva. Por último, podría aparecer una tercera fase en la que la especie queda relegada a poblaciones relictas en aquellos lugares donde las condiciones topográficas y microclimáticas locales permiten su persistencia. Sin embargo, a pesar de estas generalidades, es necesario tener en cuenta otra serie de factores como la topografía, la variabilidad interanual o las interacciones bióticas mutualistas y antagonistas que pueden alterar los efectos puramente climáticos sobre los límites de distribución de las especies.

\section{Agradecimientos}

Me gustaría agradecer a Luis Cayuela su invitación para participar en este número de Ecosistemas. Durante la escritura de este trabajo he disfrutado de una beca Marie Curie de la UE (FP7-2011IEF-300825).

\section{Referencias}

Adil, S., 2008. Climate change and forest in Morocco: case of the decay of the cedar in the Atlas Mountains. In: Poster Presentation At: International Conference "Adaptation of Forests and Forest Management to Changing Climate with Emphasis on Forest Health: A Review of Science, Policies, and Practices", Umea, Sweden: FAO/IUFRO, 25-28 August 2008.

Allen, C.D., Macalady, A.K., Chenchouni, H., Bachelet, D., McDowell, N., Vennetier, M., Kitzberger, T., Rigling, A., Breshears, D.D., Hogg, E.H., Gonzalez, P., Fensham, R., Zhang, Z., Castro, J., Demidova, N., Lim, J.H., Allard, G., Running, S.W., Semerci, A., Cobb, N. 2010. A global overview of drought and heat-induced tree mortality reveals emerging climate change risks for forests. Forest Ecology and Management 259:660-684 
Almqvist, C., Bergsten, U., Bondesson, L., Eriksson, U. 1998. Predicting germination capacity of Pinus sylvestris and Picea abies seeds using temperature data from weather stations. Canadian Journal of Forest Research 28:1530-1535.

Bale, J.S., Masters, G.J., Hodkinson, I.D., Awmack, C., Bezemer, T.M., Brown, V.K., Butterfield, J., Buse, A., Coulson, J.C., Farrar, J., Good, J.E.G., Harrington, R., Hartley, S., Jones, T.H., Lindroth, R.L., Press, M.C., Symrnioudis, I., Watt, A.D., Whittaker, J.B. 2002. Herbivory in global climate change research: direct effects of rising temperature on insect herbivores. Global Change Biology 8:1-16.

Bigler, C., Bräker, O.U., Bugmann, H., Dobbertin, M., Rigling, A., 2006. Drought as an Inciting Mortality Factor in Scots Pine Stands of the Valais, Switzerland. Ecosystems 9:330-343.

Bogino, S., Fernández Nieto, M.J., Bravo, F. 2009. Climate effect on radial growth of Pinus sylvestris at its southern and western distribution limits. Silva Fennica 43:609-623.

Cairns, D. M., Mohen, J. 2004. Herbivory influences tree lines. Journal of Ecology 92:1019-1024

Castro, J., Zamora, R., Hódar, J.A., Gómez, J.M. 2004. Seedling establishment of a boreal tree species (Pinus sylvestris) at its southernmost distribution limit: consequences of being in a marginal Mediterranean habitat. Journal of Ecology 92:266-277.

Cech, T., Tomiczek, C., 1996. Zum Kiefernsterben in Niedero sterreich. Forstschutzaktuell 17/18:12-13.

Grace, J., Berninger, F., Nagy, L. 2002. Impacts of climate change on the treeline. Annals of Botany 90:533-544.

Dobbertin, M., Wermelinger, B., Bigler, C., Bürgi, M., Carron, M., Forster, B., Gimmi, U., Rigling, A. 2007. Linking increasing drought stress to scots pine mortality and bark beetle infestations. Scientific World Journal 7:231-239

El Abidine, A.Z., 2003. Forest decline in Morocco: causes and control strategy. Science et changements planetaires/Secheresse 14:209-218.

Fabre, B., Piou, D., Desprez-Loustau, M., Marçais, B. 2011. Can the emergence of pine diplodia shoot blight in France be explained by changes in pathogen pressure linked to climate change? Global Change Biology 17:3218-3227

Fisher, M., Gardner, A.S., 1995. The status and ecology of a Juniperus-excelsa subsp. Polycarpos woodland in the northern mountains of Oman. Vegetatio 119:33-51.

Gómez-Aparicio, L., Zamora, R., Gómez, J.M., Hódar, J.A., Castro, J., Baraza, E. 2004. Applying plant facilitation to forest restoration: a metaanalysis of the use of shrubs as nurse plants. Ecological Applications 14:1128-1138.

Hampe, A., Jump, A.S. 2011. Climate relicts: past, present, future. Annual Review in Ecology, Evolution and Systematics 42:313-333.

Hampe, A., Petit, R.J. 2005. Conserving biodiversity under climate change: the rear edge matters. Ecology Letters 8:461-467.

Harsch, M.A., Hulme, P.E.McGlone, M.S., Duncan, R.P. 2009. Are treelines advancing? A global meta-analysis of treeline response to climate warming. Ecology letters 12:1040-1049.

Herrero, A., Zamora, R., Castro, J., Hódar, J.A. 2012. Limits of pine forest distribution at the treeline: Herbivory matters. Plant Ecology 213:459-469.

Hódar, J.A., Castro, J., Zamora, R. 2003. Pine processionary caterpillar Thaumetopoea pityocampa as a new threat for relict Mediterranean Scots pine forests under climatic warming. Biological Conservation 110:123-129.

Hódar, J.A., Zamora, R., Cayuela, L. 2012. Cambio climático y plagas: algo más que el clima. Ecosistemas 21(3):73-78.

Holtmeier, F.K., Broll, G. 2005. Sensitivity and response of northern hemisphere altitudinal and polar treelines to environmental change at landscape and local scales. Global Ecology and Biogeography 14:395-410.

Holtmeier, F.K., Broll, G. 2011. Response of scots pine (Pinus sylvestris) to warming climate at its altitudinal limit in northernmost subarctic Finland. Arctic 64:269-280.

Hughes, L. 2000. Biological consequences of global warming: Is the signal already apparent? Trends in Ecology and Evolution 15:56-61.

IPCC. 2007. Climate change, 2007. The Physical Science Basis: Working Group I Contribution to the Fourth Assessment Report of the IPCC. Cambridge University Press, Cambridge. UK.

Jump, A.S., Mátyás, C., Peñuelas, J. 2009. The altitude-for-latitude disparity in the range retractions of woody species. Trends in Ecology and EvoIution 24:694-701.

Juntunen, V., Neuvonen, S. 2006. Natural regeneration of Scots pine and Norway spruce close to the timberline in northern Finland. Silva Fennica 40:443-458
Juntunen, V., Neuvonen, S., Norokorpi, Y., Tasanen, T. 2002. Potential for timberline advance in northern Finland, as revealed by monitoring during 1983-99. Arctic 55:348-361.

Körner, C. 1999. Alpine plant life. Springer-Verlag, Heidelberg, Germany.

Körner, C. 2012. Alpine treelines. Functional ecology of the global high elevation tree limits. Springer, Basel. Swistzerland,

Körner, C., Sarris, D., Christodoulakis, D., 2005. Long-term increase in climatic dryness in the East-Mediterranean as evidenced for the island of Samos. Regional Environmental Change Journal 5:27-36.

Kullman, L. 1990. Dynamics of altitudinal tree-limits in Sweden: a review. Norsk geografisk Tidsskrift 44:103-116.

Kullman, L. 2002. Rapid recent-margin rise of tree and shrub species in the Swedish Scandes. Journal of Ecology 90:68-77.

Kullman, L. 2007. Tree line population monitoring of Pinus sylvestris in the Swedish Scandes, 1973-2005: implications for tree line theory and climate change ecology. Journal of Ecology 95:41-52.

Kullman, L. 2010. A richer, greener and smaller Alpine World: Review and projection of warming-induced plant cover change in the Swedish Scandes. Ambio 39:159-169.

Kullman , L., Öberg, L. 2009. Post-Little Ice Age tree line rise and climate warming in the Swedish Scances: a landscape ecological perspective. Journal of Ecology 97:415-429.

Linares, L., Pazo Sarria, R., Taïqui, L., Camarero, J.J., Ochoa, V., Lechuga, V., Seco, J.I., Viñegla, B., Sangüesa, G., Gilarte, P., Merino, J., Carreira, J.A. 2012. Efectos de las tendencias climáticas y la degradación del hábitat sobre el decaimiento de los cedrales (Cedrus atlantica) del norte de Marruecos. Ecosistemas 21(3):7-14.

Lloyd, A.H., Fastie, C.L. 2002. Spatial and temporal variability in the growth and climate response of treeline trees in Alaska. Climatic Change 52:481-509.

MacDonald, G.M., Szeicz, J.M., Claricoates, J., Dale, K.A. 1998. Response of the central Canadian treeline to recent climatic changes. Annals of the Association of American Geographers 88:183-208.

Mäkinen H, Nöjd P, Mielikäinen K., 2000. Climatic signal in annual growth variation of Norway spruce (Picea abies) along a transect from central Finland to the Arctic timberline. Canadian Journal of Forest Research 30:769-777.

Martínez-Vilalta, J., López, B.C., Loepfe, L., Lloret, F. 2012a. Stand- and tree-level determinants of the drought response of Scots pine radial growth. Oecologia168:877-888.

Martínez-Vilalta, J., Aguadé, D., Banqué, M., Barba, J., Curiel Yuste, J., Galiano, L., Garcia, N., Gómez, M.,Hereş, A.M., López, B.C., Lloret, F., Poyatos, R., Retana, J., Sus, J., Vayreda, J., Vilà-Cabrera, A. 2012b. Las poblaciones ibéricas de pino albar ante el cambio climático: con la muerte en los talones. Ecosistemas 21(3):15-21.

Mathisen, I.E., Hofgaard, A. 2011. Recent height and diameter growth variation in Scots pine (Pinus sylvestris L.) along the arctic margin: The importance of growing season versus non-growing season climate factors. Plant Ecology and Diversity 4:1-11.

Matías, L., Jump, A.S. 2012. Interactions between growth, demography and biotic interactions in determining species range limits in a warming world: the case of Pinus sylvestris. Forest Ecology and Management 282:10-22.

Matías, L., Zamora, R., Castro, J. 2012. Rare rainy events are more critical than drought intensification for woody recruitment in Mediterranean mountains: a field experiment simulating climate change. Oecologia 169:833-844.

Mendoza, I., Gómez-Aparicio, L., Zamora, R., Matías, L. 2009. Recruitment limitation of forest communities in a degraded Mediterranean landscape. Journal of Vegetation Science 20:367-376.

Meshinev, T., Apostology, I. Koleva, E. 2000. Influence of warming on timberline rising: a case study of Pinus peuce Griseb. in Bulgaria. Phytocoenologia 30:431-438.

Millar, C.I., Westfall, R.D., Delany, D.L., 2007. Response of high-elevation limber pine (Pinus flexilis) to multiyear droughts and 20th-century warming, Sierra Nevada, California, USA. Canadian Journal of Forest Research-Revue Canadienne De Recherche Forestiere 37:25082520.

Minerbi, S., 1993. Wie gesund sind unsere Walder? 10. Bericht uber den Zustand der Walder in Su dtirol. Agrar- und Forstbericht, Autonome Provinz Bozen, Assessorate fur Land-und Forstwirtschaft, pp 40.

Navarro-Cerrillo, R., Varo, M.A., Lanjeri, S., Hernandez-Clemente, R. 2007. Cartografía de defoliación en los pinares de pino silvestre (Pinus sylvestris L.) y pino salgareño (Pinus nigra Arnold.) en la Sierra de los Filabres. Ecosistemas 2007(3):163-171.

Nemani, R.R., Keeling, C.D., Hashimoto, H., Jolly, W.M., Piper, S.C., Tucker, C.J., Myneni, R.B., Running, S.W. 2003. Climate-driven increases in 
global terrestrial net primary production from 1982 to 1999 . Science 300:1560-1563.

Neuvonen, S., Niemelä, P., Virtanen, T. 1999. Climatic change and insect outbreaks in boreal forests: the role of winter temperatures. Ecological Bulletins 47:63-67.

Parmesan, C., Yohe, G. 2003. A globally coherent fingerprint of climate change impacts across natural systems. Nature 421:37-42.

Parmesan, C. 2006. Ecological and evolutionary responses to recent climate change. Annual Review of Ecology, Evolution, and Systematics 37:637669

Peñuelas, J., Boada, M. 2003. A global change-induced biome shift in the Montseny mountains (NE Spain). Global Change Biology 9:131-140.

Pohtila, E. 1980. Climatic fluctuation and forestry in Lapland. Holarctic Ecology 3:91-98.

Poupon, H., 1980. Structure et dynamique de la strate ligneuse d'une steppes Sahelienne au nord du Senegal. Travaux et documents del' O.R.S.T.O.M. 115

Rickebusch, S., Lischke, H., Bugmann, H., Guisan, A., Zimmermann, N.E. 2007. Understanding the low-temperature limitations to forest growth through calibration of a forest dynamics model with tree-ring data. Forest Ecology and Management 246:251-263.

Salminen, H., Jalkanen, R. 2007. Intra-annual height increment of Pinus sylvestris at high latitudes in Finland. Tree Physiology 27:1347-1353.

Sánchez-Salguero, R., Navarro-Cerrillo, R.M., Camarero, J.J., FernándezCancio, A., Swetnam, T.W., Zavala, M.A. 2012a. Vulnerabilidad frente a la sequía de repoblaciones de dos especies de pinos en su límite meridional en Europa. Ecosistemas 21(3):31-40.

Sánchez-Salguero, R., Navarro-Cerrillo, R.M., Swetnam, T.W., Zavala, M.A $2012 \mathrm{~b}$. Is drought the main decline factor at the rear edge of europe? the case of southern Iberian pine plantations. Forest Ecology and Management 271:158-169.

Semerci, A., Balkız, G.B., Ceylan, S., Argun, N. 2008. Examination of tree mortalities in semi-arid central Anatolian region of Turkey during last sixyear period (2002-2007). Book of Abstracts of the International Conference "Adaptation of Forests and Forest Management to Changing Climate with Emphasis on Forest Health: A Review of Science, Policies, and Practices", Umea, Sweden, FAO/IUFRO, 25-28 August 2008. p. 262.

Shiyatov, S.G. 2000. Climate dependent dynamics of the upper timberline and forest-tundra ecosystems during the last 1350 years in the polar Ural mountains, Russia. International Conference on Dendrochronology for the Third Millenium 2-7 April 2000, Mendoza, Argentina, pp. 241.

Sikström, U., Jacobson, S., Pettersson, F., Weslien, J. 2011. Crown transparency, tree mortality and stem growth of Pinus sylvestris, and colonization of Tomicus piniperda after an outbreak of Gremmeniella abietina. Forest Ecology and Management 262:2108-2119.

Slot, M., Wirth, C., Schumacher, J., Mohren, G.M.J., Shibistova, O., Lloyds, J., Ensminger I. 2005. Regeneration patterns in boreal Scots pine glades linked to cold-induced photoinhibition. Tree Physiology 25:11391150.

Stöcklin J, Körner C. 1999. Recruitment and mortality of Pinus sylvestris near the arctic tree line: the role of climatic change and herbivory. Ecological Bulletins 47:168-177.

Suarez, M.L., Ghermandi, L., Kitzberger, T., 2004. Factors predisposing episodic drought-induced tree mortality in Nothofagus: site, climatic sensitivity and growth trends. Journal of Ecology 92:954-966.

Suttle, K.B., Thomsen, M.A., Power, M.E. 2007. Species interactions reverse grassland responses to changing climate. Science 315:640-642.

Szeicz, J.M., MacDonald, G.M. 1995. Recent white spruce dynamics at the subarctic alpine treeline of north-western Canada. Journal of Ecology 83:873-885.

Thabeet, A., Vennetier, M., Gadbin-Henry, C., Denelle, N., Roux, M., Caraglio, Y., Vila, B. 2009. Response of Pinus sylvestris L. to recent climate change in the French Mediterranean region. Trees, Structure and Functions 23:843-853.

Tylianakis, J.M., Didham, R.K., Bascompte, J., Wardle, D.A. 2008. Global change and species interactions in terrestrial ecosystems. Ecology Letters 11:1351-1363.

Van der Putten, W.H., Macel, M., Visser, M.E. 2010. Predicting species distribution and abundance responses to climate change: why it is essential to include biotic interactions across trophic levels. Philosophical Transactions of the Royal Society B 365:2025-2034.

van Mantgem, P.J., Stephenson, N.L., 2007. Apparent climatically induced increase of tree mortality rates in a temperate forest. Ecology Letters 10: 909-916.

Vennetier, M., Vila, B., Liang, E.Y., Guibal, F., Thabeet, A., Gadbin-Henry, C. 2007. Impact of climate change on pine forest productivity and on the shift of a bioclimatic limit in a Mediterranean area. En: Leone V., Lovreglio R. (eds.). Proceedings of the International Workshop, MEDPINE 3, "Conservation, Regeneration and Restoration of Mediterranean Pines and their Ecosytems, Bari (Italy), 26-30 september 2005, pp. 189-197.

Walther, G.R. 2003. Plants in a warmer world. Perspectives in Plant Ecology, Evolution and Systematics 6:169-185.

Walther, G.R. 2010. Community and ecosystem responses to recent climate change. Philosophical Transactions of the Royal Society B 365:20192024.

Wardle, P., Coleman, M.C. 1992. Evidence for rising upper limits of four native New Zealand forest trees. New Zealand Journal of Botany 30:303314

Wermelinger, B., Rigling, A., Schneider, M., Dobbertin, M., 2008. Assessing the role of bark- and wood-boring insects in the decline of Scots pine (Pinus sylvestris) in the Swiss Rhone valley. Ecological Entomology 33:239-249.

Woodward, F.I. 1987. Climate and Plant Distribution. Cambridge University Press, Cambridge. 